\title{
Extension of Tycho catalog for low-extinction windows in the galactic bulge
}

\author{
T.P. Dominici, R. Teixeira, J.E. Horvath, G.A. Medina Tanco, and P. Benevides-Soares \\ Instituto Astronomico e Geofísico, Universidade de São Paulo, Av. Miguel Stéfano 4200, São Paulo, SP 04301-904, Brazil
}

Received September 16, 1998; accepted January 7, 1999

\begin{abstract}
We present in this work secondary catalogs ${ }^{1}$ up to $m_{V \text { al }} \sim 13$ based on the Tycho reference frame $(\mathrm{ESA}$, 1997) for 12 selected low-extinction fields towards the galactic bulge. The observations have been performed with the Askania-Zeiss Meridian Circle equiped with a CCD camera, located at the Abrahão de Moraes Observatory (Valinhos, Brazil) and operated by the Institute of Astronomy and Geophysics, São Paulo University. The presented catalog, though not complete, has been designed to help in intensive search programmes (e.g. microlensing and variable searches) and therefore the selected standards have a high astrometric and photometric ( $V$ band, approximately) quality. The mean precisions obtained were $0.0018^{\mathrm{s}}$ in $\alpha, 0.013^{\prime \prime}$ in $\delta, 0.030$ for the standard deviation in magnitude and 0.0042 for the magnitude when weighted with the error bars in each night (in the mean, 42 stars for the catalog of each window).
\end{abstract}

Key words: catalogs — astrometry — Galaxy: center

\section{Introduction}

The large amount of dust and gas in the direction of the galactic bulge is known to be responsible for the large extinction which makes very difficult the observation of the bulge in several bands. However, it has long been known that the irregular distribution of dust and gas presents some "holes" where the extinction is considerably lower. The best known of these so-called windows has been found by Baade (1951) and extensively studied since then. Generally speaking, these low-extinction windows offer a

Send offprint requests to: T.P. Dominici,

e-mail: tania@orion.iagusp.usp.br

1 Tables B.1 to B.12 are only available in electronic form at the CDS via anonymous ftp to cdsarc.ustrasbg.fr (130.79.128.5) or via http://cdsweb.ustrasbg.fr/Abstract.html unique opportunity to obtain information about the bulge in the optical range. Several groups (MACHO, OGLE, for example) have been recently monitoring the bulge in search of microlensing events (see Paczyński 1996, for a review).

Since April 1997 we have been conducting a small monitoring project of 12 selected low-extinction windows (see Table 1), taken from Blanco \& Terndrup (1989) and Blanco (1988), using the recently refurbished Meridian Circle of the Abrahão de Moraes Observatory (see below). The main objective of the project is the discovery and classification of variable stars. The final goal will be to have an on-line, real time processing data to stimulate the study of potentially interesting events by other observing facilities.

Meanwhile, we have faced the problem that the total number of reference stars of the usual catalogues inside the observed low-extinction fields $\left(13^{\prime}\right.$ in declination and between 3 and 6 minutes in right ascension) is small. This posed a serious difficulty for the reduction of data and the search for variable objects. As a first step towards a comprenhensive study of the windows, we have attempted to construct dense (secondary) reference catalogues intended to be of general use. The publication of the Tycho Catalog (ESA, 1997) has added an additional motivation to our task, since the refinement of the latter beyond the completeness limit $(V \sim 11)$ was already foreseen as a useful tool for future observations.

We shall describe in the next section the selected fields. Section 3 is devoted to a presentation of the instrumental facilities and data reduction. The criteria of the selection of the set of standards is discussed in Sect. 4. A general discussion in Sect. 5, the light curves in Appendix A and the new catalogues in Appendix B closes the present work.

\section{Selection of the fields}

Table 1 summarizes the list of low-extinction windows in which we have defined a relatively dense set of standards for further use. Due to specific needs of our project, but 
Table 1. Coordinates of the 12 low-extinction windows for wich secondary catalogs based on Tycho data are presented (Blanco \& Terndrup 1989 and Blanco 1988)

\begin{tabular}{lcrrrr}
\hline Window & $\#$ & $\alpha(\mathrm{J} 2000)$ & $\delta(\mathrm{J} 2000)$ & $b\left(^{\circ}\right)$ & $l\left(^{\circ}\right)$ \\
\hline BE & 5 & 181017 & -314549 & -6.02 & 0.20 \\
BG & 7 & 181806 & -325124 & -7.99 & 0.00 \\
BJ & 10 & 184049 & -344948 & -13.10 & 0.27 \\
LA & 11 & 165657 & -524453 & -6.02 & -24.77 \\
LB & 12 & 171827 & -480540 & -6.01 & -18.96 \\
LC & 13 & 172922 & -452005 & -6.03 & -15.61 \\
LD & 14 & 174459 & -404137 & -5.99 & -10.14 \\
LI & 19 & 180448 & -334746 & -5.96 & -2.11 \\
LR & 27 & 182332 & -261016 & -5.98 & +6.54 \\
LT & 29 & 183409 & -212335 & -6.04 & +9.87 \\
LU & 30 & 185126 & -131841 & -6.03 & +21.04 \\
LV & 31 & 185737 & -102335 & -6.08 & +24.35 \\
\hline
\end{tabular}

also in view of more general applications, we have proceeded to select standard stars up to $m_{V \text { al }}=13$ (we will use " $m_{V \text { al }}$ " to designate the magnitudes obtained with the Valinhos system ( $V_{\mathrm{V}}$ filter) and " $V$ " for magnitudes in the standard Jonhson system ( $V_{\mathrm{J}}$ filter $)$ ). We should emphasize that, since the primary objective was to construct an astrometric and photometric reference set, the selected stars do not constitute a complete sample up to the stated magnitude limit. It is also clear that, due to instrumental limitations and sky quality of the site, the extension of quality observations to fainter magnitudes is not devoid of problems. However, according to the performed tests and preliminary results (Dominici 1998), we are be able to monitor variable stars up to $m_{V \text { al }}=14.5$; and construct complete stellar catalogues up to $m_{V \text { al }} \sim 15.5$, the latter limit being slightly dependent on the field declination (see below). This relatively shallow sampling naturally means that our standards do not reside in the bulge but are just foreground stars.

\section{Observations and data reduction}

The Askania-Zeiss Abrahão de Moraes Meridian Circle, operated at the IAG/USP Valinhos Observatory $(\phi=$ $\left.46^{\circ} 58^{\prime} 03^{\prime \prime}, \lambda=-23^{\circ} 00^{\prime} 06^{\prime \prime}\right)$, is a $0.19 \mathrm{~m}$ refractor instrument and $2.6 \mathrm{~m}$ focal distance. Recently, a CCD detector was installed as a part of the continuing Bordeaux Observatory-IAG/USP collaboration (Viateau et al. 1999). The capabilities of the Meridian Circle have thus been greatly increased and allowed reliable photometric (as well as the astrometric) measurements to be performed. The CCD detector Thomson 7895A installed at Valinhos has a $512 \times 512$ pixel matrix, with a pixel scale of $1.5^{\prime \prime} /$ pixel. It is cooled down to $-40^{\circ} \mathrm{C}$ by a two stage thermocouple system. The observations are performed in a drift-scanning mode, that is, the speed of charge transfer by the CCD is the same as the speed of stellar transit

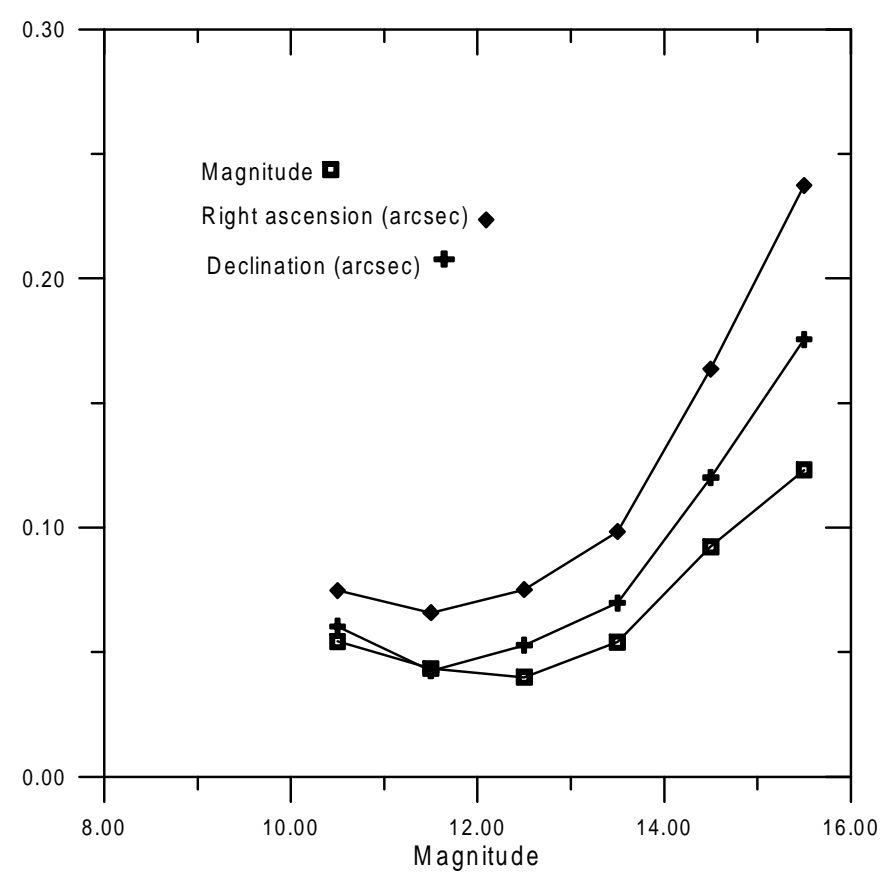

Fig. 1. Precision of the positions and magnitudes for one typical observation night

for a fixed instrument position. Therefore the integration time for a given declination $\delta$ is

$t_{\text {int }}=51 \sec \delta s$.

The observed field has a width of $13^{\prime}$ in declination by an arbitrary time in right ascension (some minutes or hours). After the installation of the CCD detector, it became possible to obtain images of several thousands of stars per night, up to magnitudes as faint as $m_{V \text { al }}=16.5$, depending on the transit time as reflected by Eq. (1).

Although the accuracy of the position and magnitude measurements depend on several factors, like the magnitude of the observed star, they have been checked to be better than 0.05 of arcsec for the position and 0.05 for the magnitude (inside the optimal magnitude interval $9<m_{V \text { al }}<14$, see Fig. 1).

The filter we have used in this and other observational programmes is somewhat wider than the standard Johnson filter $V_{\mathrm{J}}$; allowing a larger coverage towards the infrared band in order to maximize the number of objects by taking advantage of the better quantum efficiency of the CCD in that region. Figure 2 shows the response of the Valinhos filter $V_{\mathrm{V}}$ together with the standard Johnson filter $V_{\mathrm{J}}$, both curves already weighted with the CCD detector efficiency.

In order to evaluate the difference between our magnitude system and Johnson's visual band, we selected a filter (kindly provided to us by the Laboratório Nacional de Astrofísica - LNA) capable to roughly reproducing the necessary spectral characteristics to mimic the $V_{\mathrm{J}}$ band. We used this filter to perform a full observation night, aiming at several fields for wich we had good data with 


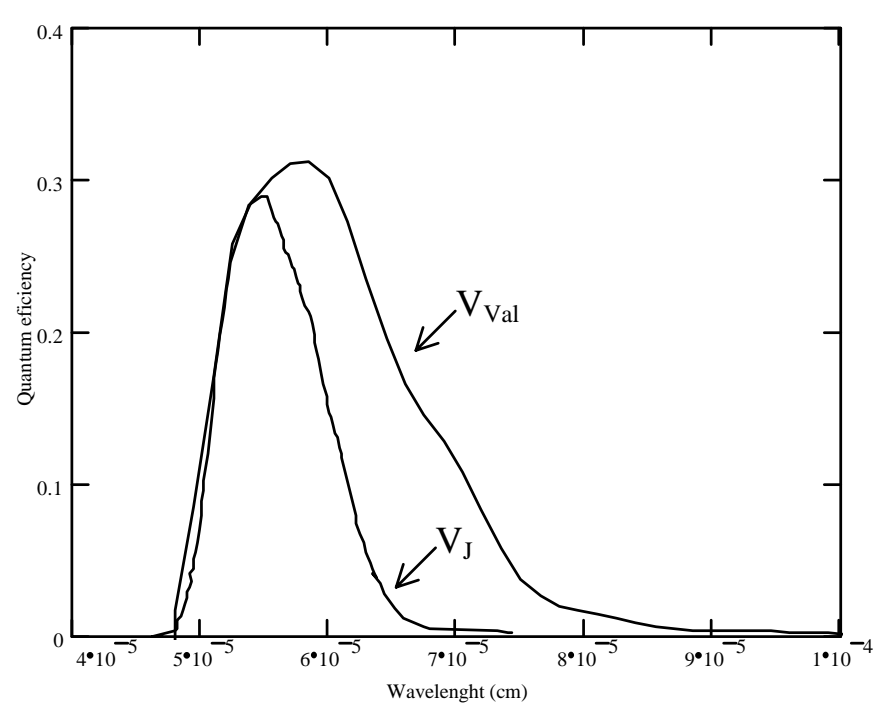

Fig. 2. The response of the Valinhos and Jonhson filters, already weighted with detector quantum efficiency

the broader filter. For the photometric reduction we made use of the Tycho catalogue $V$ magnitude.

A correlation of the magnitude difference $V-m_{V \text { al }}$ with the (poorly determined) color index $(B-V)$ as reported in the Tycho catalog is shown in Fig. 3 and can be fitted by

$V-m_{V \text { al }}=0.14-0.07(B-V)$.

However, a large scattering of the data having various origins is present and precludes a straightforward utilization of Eq. (III.2). Moreover, given the intrinsic capabilities of the Meridian Circle we are not able to measure $B$ band magnitudes properly because of focusing and detector limitations. This means that even though our claims about the photometric quality and long-term stability of the selected standards are quite secure, those stars should be recalibrated whenever other system is needed for purposes other than differential photometry. With this shortcome in mind, an approximate relationship between filters may be obtained as $m_{V \text { al }}=1.02 \mathrm{~V}-0.28$ for quick references purposes only.

To select a set of standard stars we have performed 5 to 6 observations of $\sim 1^{\mathrm{h}}$ in right ascention each, centered in the selected windows. The main objective was to include as many Tycho stars as possible in each field to link the selected set to them. In addition, 10 short observations centered around the field coordinates given in Table 1, with a total duration of 3 to 6 minutes each (this is the standard duration of the observations), were employed to construct light curves for the candidates to reference stars and to check the stability of their magnitudes.

The employed data reduction method requires a preliminar reduction, with the sky background subtracted by a linear polynomial fitted to each pixel column. Objects are identified when 3 consecutive pixels with a $2 \sigma$ confidence level are detected, where $\sigma$ is the standard deviation

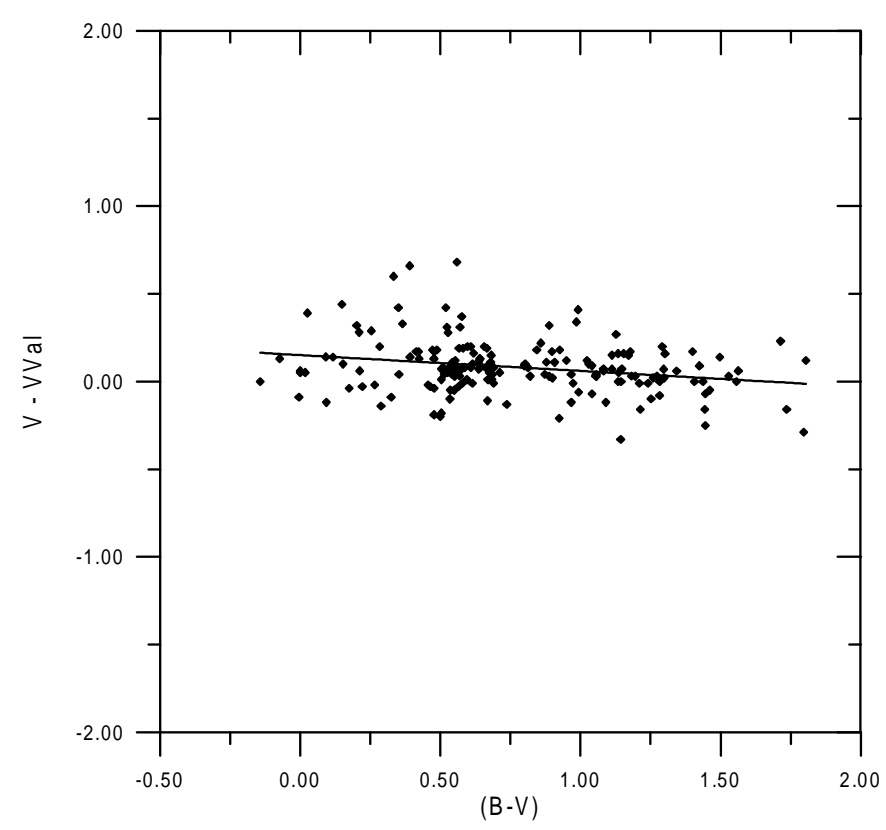

Fig. 3. Correlation of the magnitude difference $\left(V-m_{V \text { al }}\right)$ with the color index $(B-V)$

of the mean count rate in each column. A two-dimensional Gaussian curve is adjusted to the flux distribution of the objects, to obtain the $x$ and $y$ centroid coordinates, the flux and respective errors. In the following step, the celestial positions and magnitudes are calculated by a global reduction, using the field overlap among all observation nights (Eichhorn 1960; Benevides-Soares \& Teixeira 1992, and Teixeira et al. 1992). In this procedure, first of all, each night is reduced independently of the remaning data by solving the following by least squares system, with respect to reference catalogue stars:

$\alpha=a_{0}+a_{1} x+a_{2} y$

$\delta=b_{0}+b_{1} y+b_{2} x$

$m_{V \text { al }}=m_{V \text { al0 }}-2.5 \log F$

where $a_{0}$ is the initial sidereal time of the observation of the field, $b_{0}$ is the central declination of the field and $x$ and $y$ are the relative coordinates. The $x$ coordinate is measured in the diurnal motion direction while the $y$ coordinate is in the perpendicular direction. The terms $a_{1}$ and $b_{1}$ are the scale in $x$ and $y$, respectively, and $a_{2}$ and $b_{2}$ are corrections in the CCD orientation. A more robust model for the magnitude computation, using color index reported in Tycho catalog, are in development, but our tests demonstrated that actual results are confiable.

After this first reduction, the system of Eqs. (3-5) is again solved by using an iterative process, now for all stars detected in the field. At each step of iteration, the system is solved by least squares (Benevides-Soares \& Teixeira 1992 and Teixeira et al. 1992). The process converges in a few iterations (typically less than 10 steps).

The Tycho Catalog is presented in the form of a multicolumn file in which a quality criterion is given for 

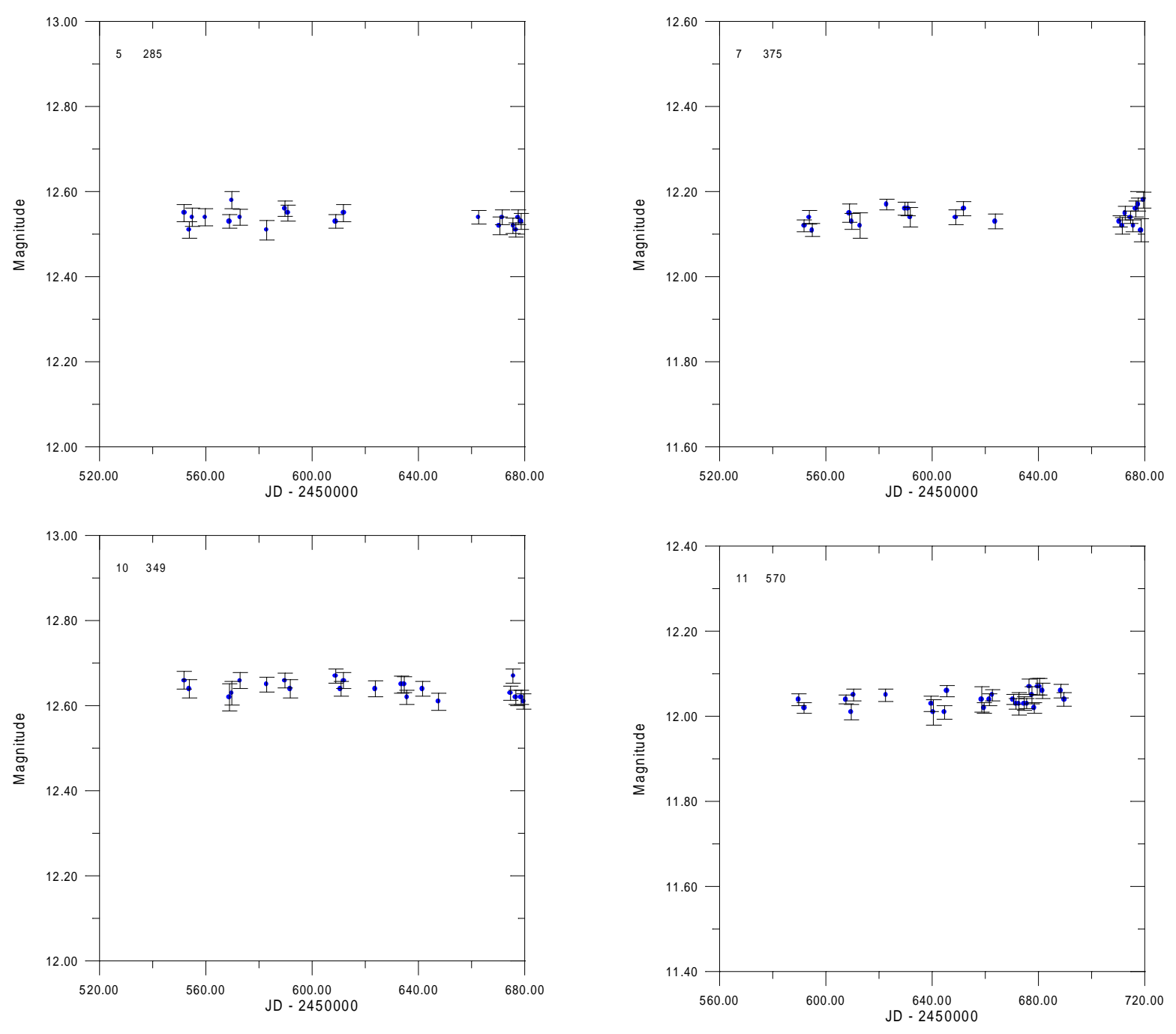

Fig. 4. Examples of light curves of the catalog stars in the windows BE, BG, BJ e LA

each object. This criterion guides the further utilization of the stars as standards for any purpose. We have concluded that the construction of secondary catalogues referred to Tycho stars of quality index worse than 5 can compromise the desired accuracy, and therefore we excluded such Tycho stars. Stars brighter than $m_{V \text { al }}=8.5$ have been also excluded because they have a saturated image in the CCD and their use would spoil the magnitude measurement of all the stars in the field. All the known variable stars were also taken out according to the GCVS (Khopolov et al. 1988) and NSV (Kukarkin et al. 1982) Catalogues, and the new variables found by the HIPPARCOS mission. With this criterion the final number of Tycho stars present in the "short" exposure lies between 1 and 8, and in the "long" exposures, between 31 and 89 stars.

\section{Construction of the reference catalogue}

After reduction of the "long" images, as described in the previous section, we have separated and relabeled all the stars brighter than $m_{V \text { al }}=13$ that appeared in all the observational runs. They constituted the first set of candidates for a reference catalogue. A visual inspection was performed to eliminate those stars located near the edges of the images and those with very near apparent neighbours (which may have complicated the magnitude determination and introduced errors in position coordinates as well). The number of "long" observations was certainly enough to guarantee the astrometric accuracy of the candidates, but not their photometric stability. Thus, to detect possible variables among our reference candidates, we have employed 10 "short" exposure observations (in principle obtained for the monitoring project) in addition to the "long" ones to construct the appropriate light 

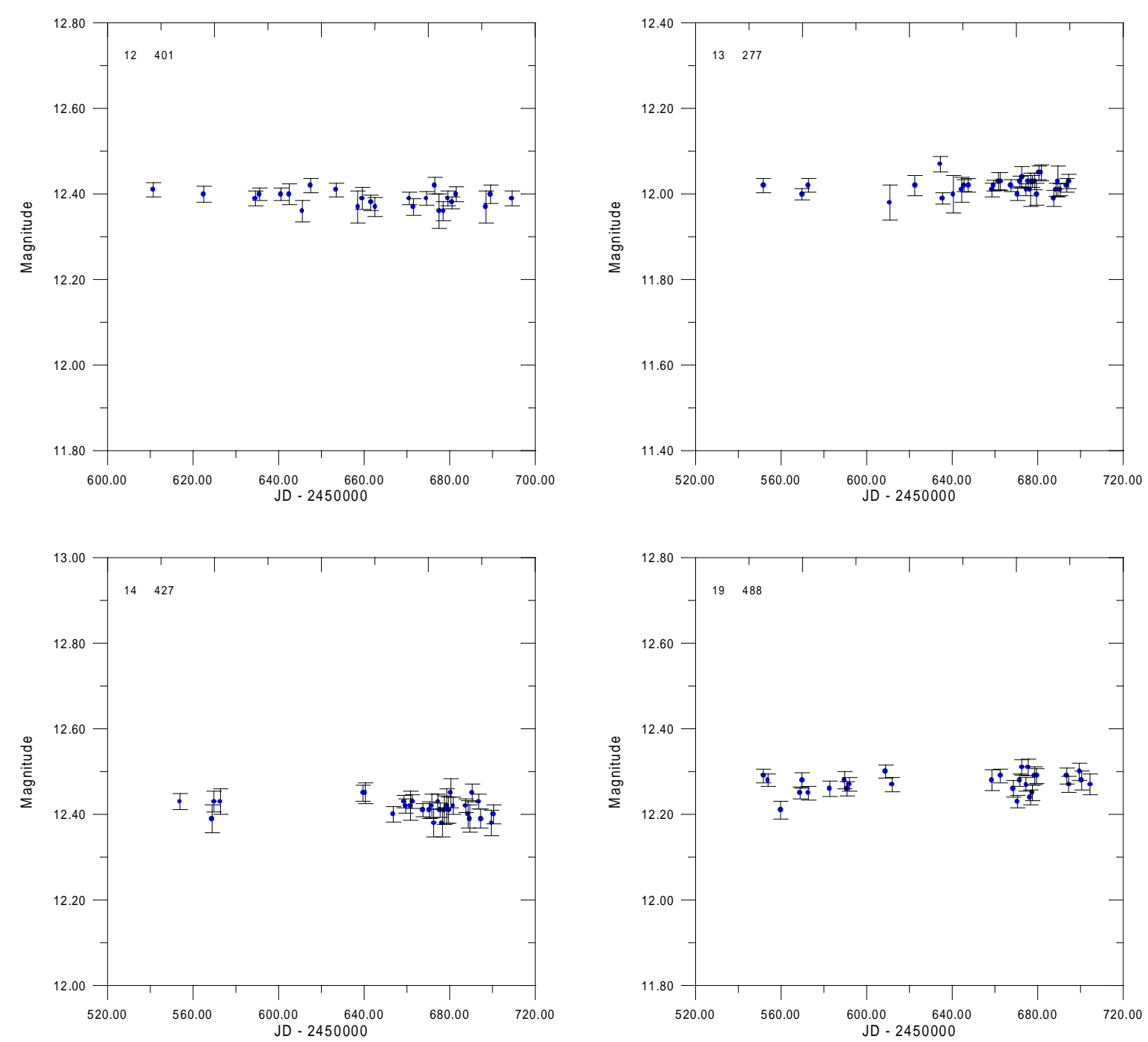

Fig. 5. Examples of light curves of the catalog stars in the windows LB, LC, LD e LI

curves. For a few fields, the "short" observations did not contain the minimum of four Tycho stars, necessary for the reduction procedure. In these cases, the number of reference stars was increased with candidates stars from the "long" observations. On the other hand, this procedure allowed us to check the sensitivity of the results to a change in the input standard stars. Once we had constructed all the light curves for the remaining candidates we proceeded to eliminate those which had large magnitude and positions variations based on the derived parameters: position and magnitude average values, their corresponding standard deviations $\left(\sigma_{\alpha}, \sigma_{\delta}, \sigma_{m_{V \text { al }}}\right)$, the average computed for the magnitude weighted with the error bars in each night and weighted error $\sigma_{\mathrm{w}}$. This selection eliminated stars having a magnitude standard deviation larger than 0.1 ; right ascention errors larger than $0.01^{\mathrm{s}}$ and declination errors larger than $0.1^{\prime \prime}$. In addition, stars for which the mean value of the magnitude was significantly different from the average weighted with the nightly error bars were also discarded (see examples of the light curves in Appendix A, Figs. 4-6). In Table 2 we present the average values of the errors in position and magnitude and the number of stars in the final catalog.

\section{Concluding remarks}

The data from standard stars appearing in this work are the result of the best quality images obtained in AprilSeptember 1997 (approximately 54 nights in total, average of 30 observations per window), reduced and processed according to the "global" procedures discussed in Sect. 2. Some stars preliminary selected as standards were in fact eliminated after the end of 1997 campaign because they did not fully satisfy the selection criteria in the long run. 

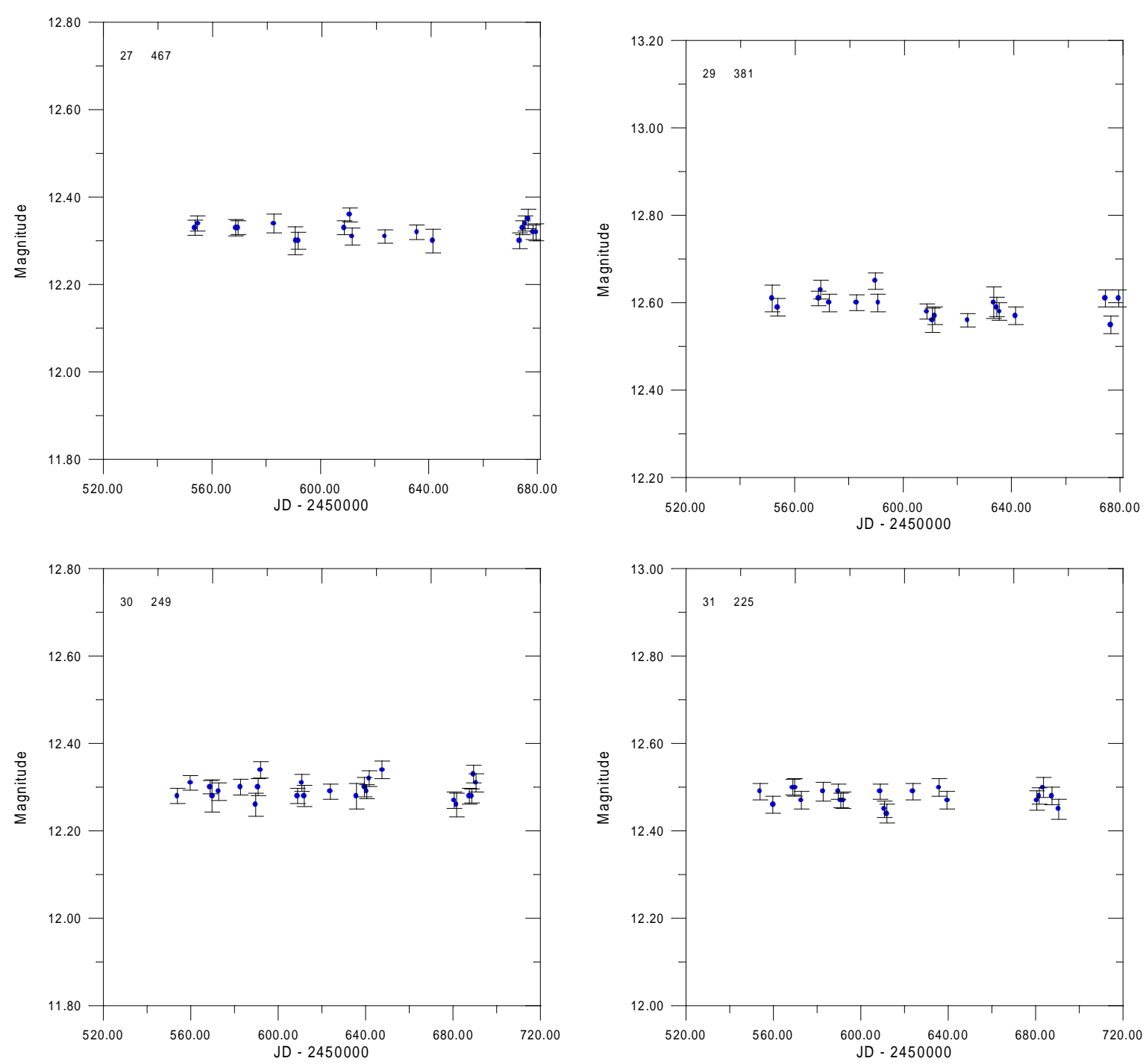

Fig. 6. Examples of light curves of the catalog stars in the windows LR, LT, LU e LV

Table 2. Average position and magnitude precisions for reference stars in the low-extinction windows. The last column indicates the number of stars in the final catalogs

\begin{tabular}{lccccc}
\hline Window & $\sigma_{\alpha}\left({ }^{\mathrm{s}}\right)$ & $\sigma_{\delta}\left({ }^{\prime \prime}\right)$ & $\sigma_{m_{V \mathrm{al}}}$ & $\sigma_{\mathrm{w}}$ & $N$ \\
\hline BE & 0.0017 & 0.015 & 0.028 & 0.0043 & 60 \\
BG & 0.0021 & 0.014 & 0.026 & 0.0040 & 41 \\
BJ & 0.0021 & 0.013 & 0.027 & 0.0041 & 43 \\
LA & 0.0019 & 0.011 & 0.035 & 0.0038 & 55 \\
LB & 0.0019 & 0.011 & 0.033 & 0.0039 & 36 \\
LC & 0.0014 & 0.011 & 0.029 & 0.0035 & 61 \\
LD & 0.0015 & 0.011 & 0.027 & 0.0036 & 47 \\
LI & 0.0010 & 0.011 & 0.031 & 0.0038 & 20 \\
LR & 0.0025 & 0.014 & 0.031 & 0.0048 & 37 \\
LT & 0.0021 & 0.014 & 0.028 & 0.0049 & 32 \\
LU & 0.0017 & 0.013 & 0.028 & 0.0045 & 31 \\
LV & 0.0015 & 0.016 & 0.034 & 0.0048 & 44 \\
\hline
\end{tabular}

We note that all these standards are being observed daily (whenever observational conditions are satisfactory) as a byproduct of our monitoring programme, and therefore the results of our catalogues are continuously being improved. Upgraded versions and the finding charts of the catalogues can be requested electronically.

Acknowledgements. We would like to thanks the financial support by FAPESP, CAPES, CNPq, Bordeaux Observatory and IAG/USP, R.D.D da Costa and the referee, F. Mignard, for useful suggestions that helped to improve the first version of the present work.

\section{Appendix A: Light curves examples}

Figures 4-6 present some examples of typical light curves accepted for the final reference set selection. 


\section{Appendix B: Valinhos reference catalogues}

The tables contain the recommended reference stars for the 12 low-extinction fields towards the galactic bulge. The first column of the catalog indicates the star label, formed by a number that designates the window (see Table 1) and a sequential counter. The following columns contain the mean right ascension, the corresponding standard deviation (in seconds); the mean declination, its standard deviation (in arcseconds) (J2000); the weighted average magnitude, its standard deviation and weighted error. Stars belonging to the Tycho Catalogue are indicated with a $T$ in the first column. We stress again that, being a natural extension of the Tycho Catalogue, the Valinhos Reference Catalogue is not complete up to the given magnitude, but instead constitude a reference set which may prove useful for dedicated studies of low-extinction fields. Complete databases up to $m_{V \text { al }}=15.5$ or better will be available soon as a product of our monitoring programme and will be published elsewhere.

\section{References}

Baade W., 1951, Publ. Obs Univ. Mich. 10, 7

Benevides-Soares P., Teixeira R., 1992, A\&A 253, 307

Blanco V.M., 1988, AJ 95, 1400

Blanco V.M., Terndrup D.M., 1989, AJ 98, 843

Dominici T.P., 1998, MSc. Thesis IAG/USP

Eichhorn H., 1960, Astron. Nachr. 285, 233

ESA SP-1200, 1997, in: The Hipparcos and Tycho catalogues

Khopolov P.N., et al., 1988, in: General Catalog of Variable Stars, Four edition, Moscow "Nauka" Publish

Kukarkin B.V., et al., 1982, in: New Suspected Variable Stars, Moscow "Nauka" Publish

Paczyński B., 1996, ARA\&A 34, 419

Teixeira R., Réquième Y., Benevides-Soares P., Rapaport M., 1992, A\&A 264, 307

Viateau B., et al., 1999, A\&AS 134, 173 